\title{
Forensic investigation of P2P cloud storage services and backbone for IoT networks: BitTorrent Sync as a case study
}

\begin{abstract}
Cloud computing can be generally regarded as the technology enabler for Internet of Things (IoT). To ensure the most effective collection of evidence from cloud-enabled IoT infrastructure, it is vital for forensic practitioners to possess a contemporary understanding of the artefacts from different cloud services and applications. In this paper, we seek to determine the data remnants from the use of the newer BitTorrent Sync applications (version 2.x). Findings from our research using mobile and computer devices running Windows, Mac OS, Ubuntu, iOS, and Android devices suggested that artefacts relating to the installation, uninstallation, log-in, log-off, and file synchronisation could be recovered, which are potential sources of IoT forensics. We also extend the cloud forensics framework of Martini and Choo to provide a forensically sound investigation methodology for the newer BitTorrent Sync applications.
\end{abstract}

Keyword: Internet of things forensics; Cloud forensics; P2p cloud investigation; Computer forensics; Mobile forensics; BitTorrent Sync 